\title{
Some Reflections on Collaborative Research in the Education of Young Scientists
}

J Bentley

Microscopy and Microanalytical Sciences, P. O. Box 7103, Oak Ridge, TN 37831-7103

For most of my career I was heavily involved in collaborative research through the Shared Research Equipment (SHaRE) program and facility at Oak Ridge National Laboratory (ORNL). Over the years I had the privilege of collaborating with many professionals from industry and academia but also with many more graduate students and post docs. Most of the time these collaborators were drawn to SHaRE because the needed equipment was not available at their home institutions. Sometimes they did not have appropriate specimen preparation equipment. Another attraction was the experience and expertise of staff at ORNL. Interestingly, SHaRE was started in 1977 by Jim Stiegler who was group leader of the radiation effects and microstructural analysis group (REMAG) with the intention of bringing in visitors to help with ongoing ORNL research. However, SHaRE soon changed to REMAG staff applying their special equipment and expertise to the projects of visitors. Several years after inception, the program was formalized with modest funding from Basic Energy Sciences (BES) at the Department of Energy (DOE) through Oak Ridge Associated Universities (ORAU) that supported a microscopist/technician (over the years a job successively filled by Jerry Lehman, Karren More and Neal Evans) and partial travel expense reimbursement for participants. Program participation and the awarding of travel funds were based on short proposals reviewed by a committee of ORNL and external professionals. Our rating system was similar to the unofficial one used in early years at Ultramicroscopy, but not many proposals fell into the category of Judith Reiffel's figure 3(c) [1]. In the new millennium, DOE managers decided that SHaRE should operate as a user facility and promote external users to work independently with less emphasis on collaborative research. A later major change was the incorporation of their surviving electron-microscopy-based user facilities at Oak Ridge, Argonne and Berkeley into larger user facilities at each respective site; SHaRE became part of the Center for Nanophase Materials Sciences (CNMS).

When SHaRE functioned as a collaborative research program and facility, most projects were for one or two visits of typically from two to five days, whereas a minority involved multi-year research efforts (with annual refereeing) often on multiple topics. Barry Carter falls in the latter category. The wideranging projects from his group included work on thin amorphous films at grain boundaries in $\mathrm{Al}_{2} \mathrm{O}_{3}$ [2]; microanalysis of a directionally solidified cobalt oxide-zirconia eutectic [3]; microstructure and chemistry of $\mathrm{MgO}$ - and $\mathrm{CoO}$-doped nickel-titanate spinels [4,5]; the secondary fluorescence correction for X-ray microanalysis in the analytical electron microscope (AEM) [6]; energy-dispersive X-ray spectroscopy (EDS) of magnesium ferrite dendrites at low voltage in a field-emission gun scanning electron microscope (FEG-SEM) [7]; microanalysis and in-situ transmission electron microscope (TEM) crystallization of anorthite glass films on $\alpha-\mathrm{Al}_{2} \mathrm{O}_{3}$ [8]; beam damage during TEM of mesoporous materials [9]; AEM of amorphous and oxide surface layers on nanoparticles [10]; electron energy-loss spectroscopy (EELS) of ceria abrasives [11]; and epitactic formation of forsterite on $\mathrm{MgO}$ single crystals during in-situ annealing [12]. Success at SHaRE was no different from at a home institution. The most important requirements were good students who knew the literature, a sound research plan with realistic goals, and good quality specimens. Techniques such as spectrum imaging by simultaneous EDS and EELS on an AEM equipped with a FEG and operated in scanning transmission (STEM) mode or elemental mapping by core-loss energy-filtered TEM (EFTEM) required mastering complex alignment and optimized acquisition protocols that were achievable only by the most capable students who made 
many visits, otherwise it was necessary for staff to take the lead in operating the instrumentation. Nowadays, more automated alignments and data acquisition help significantly, but skill in choosing areas for analysis and recognition of when things are not optimum, such as specimens being too thick or artifacts due to specimen preparation or beam damage being present, still takes time to learn.

Since my retirement from ORNL, I have been fortunate to collaborate with Jim Wittig and his students at Vanderbilt University. Work on three transformation- and twinning-induced plasticity (TRIP-TWIP) austenitic steels [Fe-22/25/28Mn-3Al-3Si (wt\%)] included the determination of stacking fault energies by weak-beam dark-field (WBDF) TEM imaging of dissociated dislocations $[13,14]$, the microstructural and strain-hardening evolution during tensile deformation [15] and the effects of high strain rates on mechanical properties and deformation microstructures [16]. Recently, work on a dual-phase mediumMn steel [Fe-12Mn-3Al-0.05C (wt\%)] has involved multi-scale characterization of austenite reversion and martensite recovery [17] with mechanical property measurements and finite-element modeling.

It has been most satisfying to mentor young scientists and humbling to be informed that the scope of their dissertation research or even professional career had been changed by exposure to microanalytical techniques not available (or sometimes not correctly implemented) at their home institutions [18].

\section{References:}

[1] J Reiffel, Ultramicroscopy 49 (1993) 443-6.

[2] Y Kouh-Simpson, CB Carter, KJ Morrisey, P Angelini and J Bentley, J Mater Sci 21 (1986) 2689-96.

[3] J Bentley, S McKernan, CB Carter and A Revcolevschi, Microbeam Analysis 2 (1993) S286-7; Inst Phys Conf Ser 138 Proc EMAG '93 (Institute of Physics, Bristol, 1993) 39-42.

[4] IM Anderson, CB Carter and J Bentley, Proc MSA (1994) 1002-3.

[5] IM Anderson, J Bentley and CB Carter, MRS Symp Proc 332 (1994) 309-14.

[6] IM Anderson, J Bentley and CB Carter, J Microscopy 178 (1995) 226-239; Ultramicroscopy 68 (1997) 77-94; Ultramicroscopy 68 (1997) 95-107.

[7] MT Johnson, IM Anderson, J Bentley and CB Carter, Proc Microsc Microanal (1996) 478-9.

[8] MP Mallamaci, J Bentley and CB Carter, Acta Mater 46 (1998) 283-303.

[9] CF Blanford, J Bentley, A Stein and CB Carter, Microsc Microanal 4(suppl 2) (1998) 712-3.

[10] CR Perrey, CB Carter, J Bentley and M Lentzen, Microsc Microanal 9(suppl 2) (2003) 412-3.

[11] SR Gilliss, J Bentley, and CB Carter, Appl Surf Sci 241 (2005) 61-67.

[12] JK Farrer, MT Johnson, J Bentley, and CB Carter, Surf Sci 587 (2005) 205-218.

[13] DT Pierce, J Bentley, JA Jiménez and JE Wittig, Scripta Mater 66 (2012) 753-6.

[14] DT Pierce, JA Jiménez, J Bentley, D Raabe, C Oskay and JE Wittig, Acta Mater 68 (2014) 238-253.

[15] DT Pierce, JA Jiménez, J Bentley, D Raabe, J.E. Wittig, Acta Mater 100 (2015) 178-190.

[16] JT Benzing, WA Poling, DT Pierce, J Bentley, KO Findley, D Raabe and JE Wittig, Mat Sci Eng A 711 (2018) 78-92.

[17] JT Benzing, A Kwiatkowski da Silva, L Morsdorf, J Bentley, D Ponge, A Dutta, J Han, JR McBride, B Van Leer, B Gault, D Raabe and JE Wittig, Acta Mater 166 (2019) 512-530.

[18] Congratulations to my friends Dave Williams and Barry Carter on their TMBA and sincere thanks to Barry for stimulating and productive collaborations over a period of several decades. My heartfelt thanks go to Barry and Bryony Carter for their wonderful hospitality during my many visits to Ithaca, Minneapolis and Connecticut, notwithstanding a humorous episode with towels at Camelot Drive, Minnetonka, and also to Jim and Eva Wittig for their amazing hospitality during my many visits to Nashville. My appointment as Adjoint Professor at Vanderbilt University is gratefully acknowledged. 